Dept. of Math. Univ. of Oslo

Pure Mathematics No. 26

ISSN 0806-2439 September 2005

\title{
THE MINIMAL ENTROPY MARTINGALE MEASURE AND NUMERICAL OPTION PRICING FOR THE BARNDORFF-NIELSEN - SHEPHARD STOCHASTIC VOLATILITY MODEL
}

\author{
FRED ESPEN BENTH AND MARTIN GROTH
}

\begin{abstract}
We develop and apply a numerical scheme for pricing options for the stochastic volatility model proposed by Barndorff-Nielsen and Shephard. This non-Gaussian OrnsteinUhlenbeck type of volatility model gives rise to an incomplete market, and we consider the option prices under the minimal entropy martingale measure. To price numerically options with respect to this risk neutral measure, one needs to consider a Black \& Scholes type of partial differential equation, with an integro-term arising from the volatility process. We suggest finite difference schemes to solve this parabolic integro-partial differential equation, and derive appropriate boundary conditions for the finite difference method. As an application of our algorithm, we consider price deviations from the Black \& Scholes formula for call options, and the implications of the stochastic volatility on the shape of the volatility smile.
\end{abstract}

\section{INTRODUCTION}

Barndorff-Nielsen and Shephard proposed in [6] to model the price dynamics of financial assets as a geometric Brownian motion where the (squared) volatility process follows a non-Gaussian Ornstein-Uhlenbeck (OU) process. This stochastic dynamics gives rise to an incomplete financial market, where there exist a continuum of risk-neutral probabilities for arbitrage-free valuation of options. Nicolato and Venardos [15] have applied structure preserving martingale measures to price European options in terms of Laplace transforms, being suitable for numerical inversion techniques. In the present paper we study the problem of pricing European options under the minimal entropy martingale measure (MEMM), and propose a numerical method for solving the associated parabolic integro-partial differential equation.

The BNS-model assumes that the squared volatility is given as an Ornstein-Uhlenbeck process reverting to zero, with the stochastic innovations given by a subordinator process. This modeling perspective has the advantage of capturing both the heavy tails and the dependency structure observed in financial return data. Furthermore, it allows for an easy way to achieve this empirically by separating the modelling of the return distribution and the autocorrelation function of the returns. The reader is directed to [6] for more details.

Based on utility indifference pricing, it is known that for an issuer of an option with exponential risk preferences, the lowest acceptable price will be given by the discounted expected payoff with respect to the MEMM. Mathematically, we reach this price as the indifference price when risk aversion tends to zero. On the other hand, this price is the

Date: September 24, 2005.

Key words and phrases. Integro-partial differential equation, Lax-Wendroff scheme, Stochastic volatility, Lévy process, minimal entropy martingale measure. 
highest acceptable price for the buyer, which gives us a rationale for choosing a pricing measure in the family of all equivalent martingale measures. In Benth and Meyer-Brandis [7] the density process for the MEMM is derived for the BNS-model, together with the associated parabolic integro-partial differential equation giving the price dynamics of options. A crucial ingredient is a function which rescales the Lévy jump measure of the subordinator process for the volatility dynamics under the MEMM, turning the Lévy dynamics into a state dependent Markov jump process.

The option price dynamics satisfies a parabolic integro-partial differential equation (integroPDE, for short) which consists of a standard Black \& Scholes operator together with a nonlocal integral operator. Discretizing this equation using finite differences, leads to the problem of finding suitable boundary conditions on the finite solution domain. Given the detailed description of the state dynamics of the price process under the MEMM, we are able to derive asymptotics for the option price which yields boundary conditions for the numerical algorithm, arbitrary far out from the solution domain. This enables us to consider the integral term even outside the solution domain, a convenient feature when considering the integralpart of the problem. We suggest to use operator splitting on the two dimensional problem and derive finite difference schemes, i.e. Lax-Wendroff schemes. For the integral-part we consider it as a source term and use a simple trapezoidal rule to numerically evaluate the integral.

Approaching the problem of pricing options by solving the associated integro-PDE allows for a simple way to consider sensitivity measures like the delta or the gamma of the option by numerical differentiation. Other methods, like inversion of Laplace transforms and Monte Carlo methods, provide us with a price only for specified values of the volatility and the underlying asset.

Using our numerical algorithm, we analyze the price difference between the Black \& Scholes formula and the MEMM option price, for comparable models. The BNS-model is supposed to be driven by an inverse Gaussian subordinator, leading to normal inverse Gaussian distributed returns, and we collected parameter estimates from Nicolato and Venardos [15]. It turns out that the difference depends crucially on the moneyness of the option, and that the Black \& Scholes price can be both greater and less than the MEMM option price. For far-out and -in-the-money options the difference is negligible, while it is crucial for options close to at-the-money. A further analysis reveals that pricing options under the MEMM produces a volatility smile.

The paper is organized as follows: In the next section we recall some background on the model and the minimal entropy martingale measure. Section three concerns the boundary conditions of the finite solution domain of the linked system of PDEs. Finite difference schemes are proposed in Section four and finally in Section five we apply the numerical solver to some test problems and discuss the results.

\section{An integro-Black \& Scholes PDE for the MEMM PRice}

Consider a market consisting of two assets, a bond and a risky asset with price processes denoted $R(t)$ and $S(t)$, respectively. We assume that the bond yields a risk-free rate of $r$, and thus has the standard price dynamics,

$$
\mathrm{d} R(t)=r R(t) \mathrm{d} t
$$

with initial value $R(0)=1$. The risky asset is evolving according to the stochastic volatility model proposed by Barndorff-Nielsen and Shephard [6], where the squared volatility is given 
by a non-Gaussian Ornstein-Uhlenbeck process:

$$
\begin{array}{r}
\mathrm{d} S(t)=(\mu+\beta Y(t)) S(t) \mathrm{d} t+\sqrt{Y(t)} S(t) \mathrm{d} B(t), \quad S(0)=s>0 \\
\mathrm{~d} Y(t)=-\lambda Y(t) \mathrm{d} t+\mathrm{d} L(\lambda t), \quad Y(0)=y>0,
\end{array}
$$

where $B(t)$ is a Brownian motion and $L(t)$ is a pure-jump subordinator (that is, an increasing pure-jump Lévy process with no drift). We let $\left\{\mathcal{F}_{t}\right\}_{t \geq 0}$ be the completion of the filtration $\sigma(B(s), L(\lambda s) ; s \leq t)$ generated by the Brownian motion and the subordinator such that $\left(\Omega, \mathcal{F}, \mathcal{F}_{t}, \mathbb{P}\right)$ becomes a complete filtered probability space. The Lévy measure of the subordinator is denoted $\ell(\mathrm{d} z)$, and satisfies by definition $\int_{0}^{\infty} \min (1, z) \ell(\mathrm{d} z)<\infty$. We impose a stronger exponential integrability condition on the Lévy measure, given by

$$
\int_{1}^{\infty}\left\{\mathrm{e}^{c z}-1\right\} \ell(\mathrm{d} z)<\infty
$$

for the constant

$$
c=\frac{\beta^{2}}{\lambda}\left(1-\mathrm{e}^{-\lambda T}\right) .
$$

Remark here that under this integrability condition, the moment generating function of $L(1)$ exists for all $|\theta| \leq c$, being defined as

$$
\mathbb{E}[\exp (\theta L(1))]=\exp (\phi(\theta))
$$

where

$$
\phi(\theta)=\int_{0}^{\infty}\left\{\mathrm{e}^{\theta z}-1\right\} \ell(\mathrm{d} z) .
$$

Benth and Meyer-Brandis [7] derived the density process of the MEMM for the stochastic volatility model described above under the exponential integrability condition (2.3). We now recall some results from their paper which will be useful in our context, and note that these results have been extended by Rheinländer and Steiger [16] to the BNS-model with leverage. Denoting the MEMM by $\mathbb{Q}_{M E}$, the density process $Z(t)$ can be represented as

$$
Z(t):=Z^{B}(t) Z^{L}(t)
$$

where $Z^{B}(t)$ and $Z^{L}(t)$ are defined as the stochastic exponentials

$$
\begin{aligned}
& Z^{B}(t)=\exp \left(-\int_{0}^{t} \frac{\mu+\beta Y(s)}{\sqrt{Y(s)}} \mathrm{d} B(s)-\int_{0}^{t} \frac{1}{2} \frac{(\mu+\beta Y(s))^{2}}{Y(s)} \mathrm{d} s\right), \\
& Z^{L}(t)=\exp \left(\int_{0}^{t} \int_{0}^{\infty} \ln \delta(Y(s), z, s) N(\mathrm{~d} z, \mathrm{~d} s)+\int_{0}^{t} \int_{0}^{\infty}(1-\delta(Y(s), z, s)) \ell(\mathrm{d} z) \mathrm{d} s\right) .
\end{aligned}
$$

Here, $N(\mathrm{~d} z, \mathrm{~d} t)$ is the Poisson random measure of $L$ and the function $\delta(y, z, t)$ is defined as

$$
\delta(y, z, t):=\frac{H(t, y+z)}{H(t, y)} .
$$

where

$$
H(t, y)=\mathbb{E}\left[\exp \left(-\frac{1}{2} \int_{t}^{T}\left\{\frac{\mu^{2}}{Y(s)}+2 \mu \beta+\beta^{2} Y(s)\right\} \mathrm{d} u\right) \mid Y(t)=y\right]
$$


for $(t, y) \in[0, T] \times \mathbb{R}_{+}$. This function will play a key role in the derivations of MEMM prices for claims, since it gives the jump characteristics of the subordinator under $\mathbb{Q}_{M E}$. In fact, the dynamics of the processes $S(t)$ and $Y(t)$ under $\mathbb{Q}_{M E}$ are given by

$$
\begin{aligned}
\mathrm{d} \widetilde{S}(t) & =\sqrt{\widetilde{Y}(t)} \widetilde{S}(t) \mathrm{d} \widetilde{B}(t), \\
\mathrm{d} \widetilde{Y}(t) & =-\lambda \widetilde{Y}(t) \mathrm{d} t+\mathrm{d} \widetilde{L}(\lambda t),
\end{aligned}
$$

where $\widetilde{B}(t)$ is a Brownian motion. The subordinator is transformed to a pure jump Markov process $\widetilde{L}(t)$, having jump measure

$$
\widetilde{\ell}(\omega, \mathrm{d} z, \mathrm{~d} t)=\frac{H(t, \tilde{Y}(t, \omega)+z)}{H(t, \widetilde{Y}(t, \omega))} \ell(\mathrm{d} z) \mathrm{d} t .
$$

We observe that the function $H(t, y)$ rescales the jumps of the subordinator process. Moreover, the jump measure becomes time-inhomogeneous and state-dependent, thus $\widetilde{L}$ is not even an independent increment (or Sato) process under the MEMM, except for the case $\mu=0$.

We find that (2.4) is the Feynman-Kac representation of the integro-PDE

$$
\partial_{t} H(t, y)-\frac{1}{2}\left(\frac{\mu^{2}}{y}+2 \mu \beta+\beta^{2} y\right) H(t, y)+\mathcal{L}_{Y} H(t, y)=0, \quad(t, y) \in[0, T) \times \mathbb{R}_{+}
$$

with

$$
\mathcal{L}_{Y} H(t, y)=-\lambda y \partial_{y} H(t, y)+\lambda \int_{0}^{\infty}\{H(t, y+z)-H(t, y)\} \ell(\mathrm{d} z),
$$

and terminal data $H(T, y)=1, y \in \mathbb{R}_{+}$. We have used the notation $\partial_{x}$ for partial differentiation with respect to the argument $x$ of a function.

In general, it is hard to derive an explicit expression for the expectation in (2.7) defining the function $H$. However, for the special case when $\mu=0$ we can derive a solution, as proved in Benth and Meyer-Brandis [7]. Since we will need this later,, we include the result here:

Lemma 2.1. Assume $\mu=0$. Then it holds,

$$
H(t, y)=\exp (b(t) y+c(t))
$$

where $b(t)$ and $c(t)$ are defined as

$$
b(t)=-\frac{\beta^{2}}{2 \lambda}(1-\exp (-\lambda(T-t))), \quad c(t)=\lambda \int_{t}^{T} \phi(b(u)) \mathrm{d} u .
$$

We proceed further to discuss the price of claims under the MEMM. Consider a contingent claim of European type with payoff $f(S(T))$ at the exercise time $T$. We suppose that $f$ is of linear growth in order to assure integrability under MEMM. Let $\Lambda(t, y, s)$ denote the minimal entropy price of the contingent claim at time $t$ conditioned on $S(t)=s$ and $Y(t)=y$,

$$
\Lambda(t, y, s)=\mathrm{e}^{-r(T-t)} \mathbb{E}_{\mathbb{Q}_{M E}}[f(S(T)) \mid Y(t)=y, S(t)=s] .
$$

Since $S(t)$ is a martingale with respect to $\mathbb{Q}_{M E}$, we easily see that the above price is welldefined due to the linear growth of $f$. We may rewrite the price as

$$
\Lambda(t, y, s)=\mathrm{e}^{-r(T-t)} \mathbb{E}[f(\widetilde{S}(T)) \mid \widetilde{Y}(t)=y, \widetilde{S}(t)=s],
$$

which is the Feynman-Kac representation of the following Black \& Scholes integral equation

$$
\partial_{t} \Lambda(t, y, s)+r s \partial_{s} \Lambda(t, y, s)+\frac{1}{2} y s^{2} \partial_{s s} \Lambda(t, y, s)+\mathcal{L}_{Y}^{\mathrm{MEMM}} \Lambda(t, y, s)=r \Lambda(t, y, s),
$$


with $(t, y, s) \in[0, T) \times \mathbb{R}_{+}^{2}$,

$$
\mathcal{L}_{Y}^{\mathrm{MEMM}} \Lambda(t, y, s)=-\lambda y \partial_{y} \Lambda(t, y, s)+\lambda \int_{0}^{\infty}(\Lambda(t, y+z, s)-\Lambda(t, y, s)) \frac{H(t, y+z)}{H(t, y)} \ell(\mathrm{d} z)
$$

and terminal condition

$$
\Lambda(T, y, s)=f(s), \quad(y, s) \in \mathbb{R}_{+} \times \mathbb{R}_{+} .
$$

We shall approach the calculation of option prices under MEMM by solving numerically the integro-PDE above. We remark that to solve for $\Lambda$, knowledge of $H$ is required, which also solves an integro-PDE. Thus, we must consider a coupled system of two integro-PDEs when calculating the option prices for the BNS-model under the MEMM.

\section{BOUNDARY CONDITIONS FOR THE INTEGRO-PDEs ON THE SOLUTION DOMAINS}

The coupled system of integro-PDEs (2.7) and (2.13) is defined on the positive half plane for both $s$ and $y$. Applying a finite difference method to solve this system numerically requires that we constrain the problem to a finite solution domain, where we must impose conditions on the solution along the boundary of the domain. Furthermore, the integral terms in both PDEs will naturally extend beyond any finite truncation of the $y$-direction, and we need to find conditions which enable us to analyze this integral also outside the solution domain. In this section we derive the necessary boundary conditions required to use finite difference methods to find $\Lambda$.

3.1. Boundary conditions for $H$. We start with deriving some asymptotic results for the function $H(t, y)$ when $y$ becomes large and small. These results will give us the correct boundary conditions when truncating the solution domain in the spatial dimension $y$.

Lemma 3.1. It holds,

$$
\lim _{y \rightarrow \infty}|H(t, y ; \mu)-H(t, y ; 0)|=0,
$$

where the notation $H(t, y ; \mu)$ is introduced in order to emphasize the dependency on $\mu$ in (2.4).

Proof. When $y \rightarrow \infty$, we see that $\mu^{2} / Y^{t, y} \rightarrow 0$ a.s.. The result holds by dominated convergence.

From this Lemma we see that for large values of $y$, we have that $H(t, y ; \mu) \approx H(t, y ; 0)$, and an explicit representation for $H(t, y ; 0)$ is given in Lemma 2.1. Thus, after truncation the solution domain in $y$ to the interval $\left[0, y_{\max }\right]$, we impose the condition $H(t, y ; \mu)=H(t, y ; 0)$ for $y \geq y_{\max }$ in the numerical approximation procedure. Observe that the asymptotics in the Lemma also gives us a condition on the integrand in the integral expression in (2.7) whenever the argument $y+z$ is outside the solution domain [0, $\left.y_{\max }\right]$.

The following holds for the case $y=0$ :

Lemma 3.2. Suppose $\mu \neq 0$. Then $H(t, 0)=0$.

Proof. We have that

$$
H(t, y) \leq c \mathbb{E}\left[\exp \left(-\frac{\mu^{2}}{2} \int_{0}^{T-t} \frac{1}{Y^{y}(s)} \mathrm{d} s\right)\right]
$$


for some positive constant $c$, where

$$
Y^{y}(s)=y \mathrm{e}^{-\lambda s}+\mathrm{e}^{-\lambda s} \int_{0}^{s} \mathrm{e}^{\lambda s} \mathrm{~d} L(\lambda u)
$$

Letting $y \downarrow 0$, we see that

$$
0 \leq \lim _{y \downarrow 0} H(t, y) \leq c \mathbb{E}\left[\exp \left(-\frac{\mu^{2}}{2} \int_{0}^{T-t} \frac{1}{Y^{0}(s)} \mathrm{d} s\right)\right],
$$

if the limit exists. We prove that the right-hand side of this expression is equal to zero, from which we can conclude the claim. This is shown by demonstrating the the integral with respect to $s$ inside the exponential is diverging to infinity. The singularity is obtained for the lower integration limit. For $\epsilon>0$ sufficiently small we have that for $s \leq \epsilon$,

$$
Y^{0}(s)=\int_{0}^{s} \mathrm{e}^{-\lambda(s-u)} \mathrm{d} L(\lambda u) \approx L(\lambda s) \quad \text { a.s. }
$$

Furthermore, from Prop. 8, p. 84 in Bertoin [8], we know that for a subordinator $L$ it holds $\lim _{t \downarrow 0} t^{-1} L(t)=d$, a.s., where $d$ is the drift of $L$. Thus, for $s \leq \epsilon$, it holds approximately

$$
\frac{1}{Y^{0}(s)}=\frac{s^{-1}}{s^{-1} Y^{0}(s)} \approx \frac{s^{-1}}{d \lambda}
$$

which is singular when integrating from zero. Thus, the Lemma holds.

In our numerical calculations, we impose the boundary condition $H(t, 0)=0$ for $t \in[0, T)$ when $\mu \neq 0$.

Note that for $\mu=0$, we find from Lemma 2.1 that

$$
H(t, 0)=\exp \left(\lambda \int_{t}^{T} \phi(b(u)) \mathrm{d} u\right)
$$

which is not equal to zero. Hence, the two cases $\mu=0$ and $\mu \neq 0$ lead to completely different boundary conditions. In most practical situations, $\mu \neq 0$, and this is also the case we shall focus on when applying our numerical solution algorithm in the next Section.

3.2. Boundary conditions for $\Lambda$. The domain of the integro-PDE (2.13) is $(t, y, s) \in$ $[0, T) \times \mathbb{R}_{+}^{2}$. Introducing a finite difference approximation, we shall consider the truncated domain $(t, y, s) \in[0, T) \times\left[0, y_{\max }\right] \times\left[0, s_{\max }\right]$, which requires conditions on the solution $\Lambda$ at the boundaries $s=0, s=s_{\max }, y=0$ and $y=y_{\max }$.

Observing that when $S(t)=s=0$, we have $S(u)=0$ for all $u \in[t, T)$. Hence, we find that

$$
\Lambda(t, y, 0)=\mathrm{e}^{-r(T-t)} f(0)
$$

which we use as a boundary condition for $s=0$ in the integro-PDE (2.13). Let us consider the boundaries $s=s_{\max }$ and $y=y_{\max }$, where the stock price and/or the volatility is large. It turns out that the MEMM price $\Lambda$ behaves like a Black \& Scholes price with time dependent volatility when the volatility becomes large. We know from the Black-Scholes framework that as the volatility tends to infinity and $S \rightarrow \infty$, the price of a European call option will converge to the stock price. Similarly, for a European put option the price of the option will approach the strike price. See Lewis [14] for a discussion of large volatility asymptotica for stochastic volatility models where the volatility is driven by Brownian motions. For large stock prices, the asymptotics of $\Lambda$ is the same as the one we would get for constant volatility, that is, the Black \& Scholes model. We give the details in the following Lemma and Proposition. 
First, let us prove the following lemma

Lemma 3.3. It holds that

$$
\lim _{y \rightarrow \infty} \frac{\int_{0}^{T} \tilde{Y}^{y}(t) \mathrm{d} t}{\int_{0}^{T} y e^{-\lambda t} \mathrm{~d} t}=1, \text { a.s. }
$$

Proof. Note that since

$$
\int_{0}^{T} \tilde{Y}^{y}(t) \mathrm{d} t=\int_{0}^{T} y \mathrm{e}^{-\lambda t} \mathrm{~d} t+\int_{0}^{T} \mathrm{e}^{-\lambda t} \int_{0}^{t} \mathrm{e}^{\lambda s} \mathrm{~d} \widetilde{L}(\lambda s) \mathrm{d} t
$$

and $\int_{0}^{t} \mathrm{e}^{\lambda s} \mathrm{~d} \widetilde{L}(\lambda s) \geq 0$, we find that $\lim _{y \rightarrow \infty} \int_{0}^{T} \widetilde{Y}^{y}(t) \mathrm{d} t=\infty$, a.s. Moreover, since

$$
\lim _{y \rightarrow \infty} \frac{H(t, y+z)}{H(t, y)}=\mathrm{e}^{b(t) z}
$$

(with $b(t)$ as in equation $(2.10))$ ), we have

$$
\lim _{y \rightarrow \infty} \tilde{\ell}(\omega, \mathrm{d} z, \mathrm{~d} t)=\mathrm{e}^{b(t) z} \ell(\mathrm{d} z) \mathrm{d} t \text {, a.s. }
$$

and the jump measure of $\widetilde{L}$ under $\mathbb{Q}_{M E}$ converges to the jump measure of a pure jump independent increment process, denoted by $\widehat{L}$. It therefore holds that $\lim _{y \rightarrow \infty} \widetilde{L}(t)=\widehat{L}(t)$, a.s. Thus, by dominated convergence we find

$$
\frac{1}{y} \int_{0}^{t} \mathrm{e}^{\lambda s} \mathrm{~d} \widetilde{L}(\lambda s) \leq \frac{1}{y} \mathrm{e}^{\lambda t} \widetilde{L}(\lambda t) \rightarrow 0, \text { a.s. }
$$

when $y \rightarrow \infty$. Hence, we conclude that

$$
\frac{\int_{0}^{T} \widetilde{Y}^{y}(t) \mathrm{d} t}{\int_{0}^{T} y \mathrm{e}^{-\lambda t} \mathrm{~d} t}=1+\frac{\int_{0}^{T} \mathrm{e}^{-\lambda t} \frac{1}{y} \int_{0}^{t} \mathrm{e}^{\lambda s} \mathrm{~d} \widetilde{L}(\lambda s) \mathrm{d} t}{\int_{0}^{T} \mathrm{e}^{-\lambda s} \mathrm{~d} s} \rightarrow 1 \quad \text { a.s. }
$$

and the Lemma follows.

We find the following asymptotics for $\Lambda$ when $y \rightarrow \infty$ :

Proposition 3.4. We have

$$
\lim _{y \rightarrow \infty} \frac{\Lambda(t, y, s)}{\Lambda^{B S}\left(t, s ; \sigma_{t, T}^{2}(y)\right)}=1
$$

where $\Lambda^{B S}\left(t, s ; \sigma^{2}\right)$ is the Black $\&$ Scholes price for an option with payoff function $f$ written on an underlying having volatility $\sigma$, and

$$
\sigma_{t, T}^{2}(y)=\frac{y}{\lambda(T-t)}\left(1-e^{-\lambda(T-t)}\right) .
$$

Proof. The jump process $\widetilde{L}$ and the Brownian motion $\widetilde{B}$ are independent under $\mathbb{Q}_{M E}$, and we can express the option price as an integral with respect to the density of the integrated variance as follows (see Hull and White [11] and Nicolato and Venardos [15]):

$$
\Lambda(t, y, s)=\int_{0}^{\infty} \Lambda^{\mathrm{BS}}(t, s ; x / T-t) q_{\mathrm{ME}}(x) \mathrm{d} x,
$$


where $q_{\mathrm{ME}}$ is the density of $\int_{t}^{T} Y^{t, y}(s) \mathrm{d} s$ under the MEMM. Rewriting this in terms of the density of

$$
\frac{\int_{t}^{T} Y^{t, y}(s) \mathrm{d} s}{\int_{t}^{T} y \exp (-\lambda(s-t)) \mathrm{d} s}
$$

which we denote by $\widetilde{q}_{\mathrm{ME}}$, we get

$$
\Lambda(t, y, s)=\int_{0}^{\infty} \Lambda^{\mathrm{BS}}\left(t, s ; x \sigma_{t, T}^{2}\right) \widetilde{q}_{\mathrm{ME}}(x) \mathrm{d} x .
$$

Observe that by Lemma 3.3 we have that

$$
\widetilde{q}_{\mathrm{ME}}(x) \mathrm{d} x \rightarrow \delta_{1}(\mathrm{~d} x)
$$

when $y \rightarrow \infty$, where $\delta_{1}$ is the Dirac measure concentrated at 1 . Hence, we find

$$
\begin{aligned}
\frac{\Lambda(t, y, s)}{\Lambda^{\mathrm{BS}}\left(t, s ; \sigma_{t, T}^{2}\right)} & =\int_{0}^{\infty} \frac{\Lambda^{\mathrm{BS}}\left(t, s ; x \sigma_{t, T}^{2}\right)}{\Lambda^{\mathrm{BS}}\left(t, s ; \sigma_{t, T}^{2}\right)} \widetilde{q}_{\mathrm{ME}}(x) \mathrm{d} x \\
& =\int_{0}^{\infty} \frac{\Lambda^{\mathrm{BS}}\left(t, s ; x \sigma_{t, T}^{2}\right)}{\Lambda^{\mathrm{BS}}\left(t, s ; \sigma_{t, T}^{2}\right)}\left\{\widetilde{q}_{\mathrm{ME}}(x) \mathrm{d} x-\delta_{1}(\mathrm{~d} x)\right\}+1 .
\end{aligned}
$$

The first integral term converges to zero when $y \rightarrow \infty$ since the ratio

$$
\frac{\Lambda^{B S}\left(t, s ; \sigma_{t, T}^{2} x\right)}{\Lambda^{B S}\left(t, s ; \sigma_{t, T}^{2}\right)}
$$

can be bounded and the signed measure $\widetilde{q}_{\mathrm{ME}} \mathrm{d} x-\delta_{1}(\mathrm{~d} x)$ tends to zero. Hence, the Proposition follows.

The proposition above yields that for large values of $y, \Lambda$ is given by the Black \& Scholes price when the underlying asset has a time dependent volatility given by $\sqrt{y} \exp (-\lambda t / 2)$. For example, if we consider a call option, this price can be explicitly calculated, as stated in the next Corollary:

Corollary 3.5. Assume $f(x)=x-K$. Then $\Lambda^{B S}$ defined in Prop. 3.4 is given by the Black 8 Scholes pricing formula for a call option at time $t$ written on an asset with price $s$ and volatility $\sigma_{t, T}(y)$.

The knowledge of the asymptotic behaviour of $\Lambda$ in $y$ permits us to consider the integral term in the integro-PDE (2.13) for values of $y$ also outside of the solution domain. Hence, we do not need to truncate the integral term in any unnatural way when we are close to the boundary of the solution domain in the $y$-direction.

The next Proposition states the asymptotic behaviour when $s \rightarrow \infty$ :

Proposition 3.6. Suppose that $f(s) / s \rightarrow c$ for some constant $c$ when $s \rightarrow \infty$. Then

$$
\lim _{s \rightarrow \infty} \frac{\Lambda(t, y, s)}{s}=c
$$

Proof. We have by dominated convergence

$$
\frac{\Lambda(t, y, s)}{s}=\mathbb{E}_{\mathbb{Q}_{\mathrm{ME}}}\left[\frac{f\left(S^{t, s, y}(T)\right)}{S^{t, s, y}(T)} S^{t, 1, y}(T)\right] \rightarrow c \mathbb{E}_{\mathrm{ME}}\left[S^{t, 1, y}(T)\right]=c,
$$

when $s \rightarrow \infty$. 
We now finish our study of the boundary behaviour of the solution $\Lambda$ with analyzing the case $y=0$. First we note that the process $\widetilde{Y}$ is not defined for the initial state $y=0$, since the jump measure explodes. Indeed, what we observe from a heuristic point of view is that the closer we are to $y=0$, the greater the ration $H(t, y+z) / H(t, y)$ becomes, and thus the stronger the process will be pushed away from this state. A reflecting boundary at $y=0$ would imply a no-flow condition on $\Lambda$ at this boundary, i.e. the Neumann condition that the derivative of $\Lambda$ in the direction of $y$ vanishes at $y=0$. To gain further understanding of the boundary behaviour of $\Lambda$ at $y=0$, consider the following heuristic argument: Suppose that $\Lambda$ is analytical in $y$, having a series expansion

$$
\Lambda(t, y+z, s)-\lambda(t, y, s)=\sum_{n=1}^{\infty} \frac{\partial_{y}^{n} \Lambda(t, y, s)}{n !} z^{n}, n \geq 1 .
$$

Inserting this into the integro-PDE (2.13), we see that the integral part will be a sum of terms like

$$
\frac{\partial_{y}^{n} \Lambda(t, y, s)}{n ! H(t, y)} \int_{0}^{\infty} z^{n} H(t, y+z) \ell(\mathrm{d} z)
$$

Since $H(t, y) \rightarrow 0$ when $y \downarrow 0$, we must have that $\partial_{y}^{n} \Lambda(t, y, s) \rightarrow 0$, otherwise the integral terms will diverge. Hence, all the derivatives of $\Lambda$ should vanish at the boundary $y=0$, which shows the strong reflection at $y=0$ of the volatility process under the MEMM. When considering the numerical solution, we impose the condition that the derivatives up to a certain order vanish at the boundary, the simplest choice being a Neumann condition at the boundary $y=0$, that is,

$$
\partial_{y} \Lambda(t, 0, s)=0 .
$$

Such a choice may be defended by the work of Barles et.al. [3, 4, 5], which have analyzed the sensitivity of boundary conditions related to finance problems. They found that artificial boundary conditions have negligible impact on the solution outside a boundary layer. This means that even wrongly stated conditions may be smoothed out when moving into the solution domain of interest. In our case we have weakened the strong analyticity condition, but believe that the true level of volatility is sufficiently far away from $y=0$ that the impact is relatively small. Note also that we do not have exact information about the solution for $s$ and $y$ being large, requiring similar considerations for defending the appropriateness of the numerical boundary conditions.

Based on the derived boundary conditions for the two integro-PDE problems, we now move on to develop a finite difference scheme appropriate for our equations.

\section{Derivation of Finite DifFEREnCE SChemes}

In order to calculate the option price we need to solve (2.13) using a numerical method. Applying the finite difference method we derive numerical schemes, which involves truncating the infinite solution domain and solve the problem on an appropriate grid. We also have to numerically approximate the involved integral, possibly involving a Lévy measure with a singularity at zero. Let us first concentrate on the case with $r=0$.

The function $H(t, y)$ appears as a measure change in the integral part of the integro-PDE for the option price. Hence, we need to solve (2.7) first, in order to arrive at the correct option price. The non-local integral term in (2.7) need to be numerically approximated with the information attainable. Since we only know the value of $H(t, y)$ at the grid points we use 
a trapezoid integration scheme and treat the integral as a fully explicit source term. However, if we use only the points in the grid we get less points to integrate over as we get closer to the boundary $y=y_{\max }$. The approximation of the integral would then be less accurate for large $y$, which is a undesirable feature. By adding extra points in $y$ and assigning Black \& Scholes prices beyond $y_{\max }$, in accordance with Proposition 3.4, we can make sure we get a coherent treatment of the integral. If the number of extra points $n$ is large enough the decay of the measure will make sure we capture the influence from the integral. It will then be unnecessary to integrate over more than $n$ points anywhere. Reducing the number of integration points this way gives a clear speed up.

To solve the integro-PDE (2.7) we derive an implicit Lax-Wendroff scheme

$$
\begin{aligned}
\frac{R}{2}\left(-1+\frac{\lambda \Delta \tau}{2}+g \Delta \tau\right. & -R) H_{k-1}^{n+1}+\left(1+R^{2}-\frac{a g_{y} \Delta \tau^{2}}{2}+g \Delta \tau\right) H_{k}^{n+1} \\
& +\frac{R}{2}\left(1-\frac{\lambda \Delta \tau}{2}-g \Delta \tau-R\right) H_{k+1}^{n+1}=H_{k}^{n}+F_{k}^{n}
\end{aligned}
$$

where $R=\lambda y_{k} \Delta \tau / \Delta y, a=\lambda y$ and $F_{k}^{n}$ is the integral term. Furthermore, $g$ is the function

$$
g(y)=\frac{1}{2}\left(\frac{\mu^{2}}{y}+2 \mu \beta+\beta^{2} y\right)
$$

and $g_{y}$ the derivative of this function with respect to $y$.

We now turn our attention to equation (2.13). Since the option pricing is a problem in two spatial dimensions we use Gudonov dimensional splitting [10] and following Strang [17] we approximate the exact solution operator by successive use of one-dimensional operations, i.e.

$$
S(T) \Lambda_{0} \approx\left[S^{s}\left(\frac{\Delta t}{2}\right) S^{y}(\Delta t) S^{s}\left(\frac{\Delta t}{2}\right)\right]^{n} \Lambda_{0}
$$

Here $S(T)$ is the exact solution operator of (2.13), approximated by one-dimensional operators, $S^{s}(t)$ and $S^{y}(t)$, and we iterate over $n$ time steps. Since we treat the integral as a non-homogeneous term and we integrate over $y$, it seems natural to include the integral operator $\mathcal{L}_{Y}^{\mathrm{MEMM}}$ in $S^{y}$. More information about dimensional splitting for conservation laws can be found in Kröner [13].

The modeling of the volatility will in most situations result in an infinite activity Lévy process, having a singularity in the jump measure at zero. We run into numerical difficulties if we try to numerically integrate around zero in such cases. Hence, we need to start the integration at the first grid point larger than zero. Some of the pure jump Lévy process we want to consider is dominated by the small jumps and a cut-off of the integral close to zero may lead to a loss of significant parts of the integral. To make up for this we approximate parts of the integral term by a drift in the integro-PDE for the price: Letting $\epsilon$ be the first grid point larger than zero we do the approximation

$$
\int_{0}^{\epsilon}(\Lambda(t, y+z, s)-\Lambda(t, y, s)) \frac{H(t, y+z)}{H(t, y)} \ell(\mathrm{d} z) \approx \xi(t, y) \Lambda_{y}(t, y, s)
$$

where

$$
\xi(t, y)=\int_{0}^{\epsilon} z \frac{H(t, y+z)}{H(t, y)} \ell(\mathrm{d} z)
$$

The Lévy measure integrates $z$ close to zero, thus the integral makes sense. However, we need to calculate $\xi$ numerically, which we now discuss. 
Since we only have knowledge of the integrand at the grid points, there are only the two end points available for numerical integration of $\xi$. To work around this problem we assume that $H(t, y)$ is close to linear between two grid points. Then we can use linear interpolation between the points of the grid and evaluate the integrand in an arbitrary number of points. However, we still need to avoid zero because of the singularity.

If we include the terms introduced by the risk free rate of return $r>0$ in the $S^{s}$-operator, we get the Black \& Scholes PDE with Dirichlet boundary conditions. Using transformation to dimensionless parameters we can always reduce this to the heat equation. We decide to use the simple implicit finite difference scheme, here illustrated in the case $r=0$ in which case the equation is reduced to the heat equation immediately:

$$
\frac{\Lambda_{k, l}^{n+1}-\Lambda_{k, l}^{n}}{\Delta t}-\frac{\sigma^{2}\left(y_{k}\right) s_{l}^{2}}{2}\left(\frac{\Lambda_{k, l+1}^{n+1}-2 \Lambda_{k, l}^{n+1}+\Lambda_{k, l-1}^{n+1}}{\Delta s^{2}}\right)=0 .
$$

where $\Lambda_{k, l}^{n}$ is $\Lambda(t, y, s)$ evaluated at the point $(n, k, l)$ in the $(t, y, s)$ grid. For the solution operation $S^{y}$ we use the same approach as for $H(t, y)$ to derive a non-homogeneous LaxWendroff scheme: Let $a=a(y)=\lambda y$ and $\Lambda_{k, l}^{n}$ as above. A Lax-Wendroff scheme is then

$$
\frac{R}{2}\left(-1+\frac{\lambda \Delta t}{2}-R\right) \Lambda_{k-1, l}^{n+1}+\left(1+R^{2}\right) \Lambda_{k, l}^{n+1}+\frac{R}{2}\left(1-\frac{\lambda \Delta t}{2}-R\right) \Lambda_{k+1, l}^{n+1}=\Lambda_{k, l}^{n}+F_{k, l}^{n}
$$

with $R=\lambda y_{k} \Delta \tau / \Delta y$ and $F_{k, l}^{n}$ is the integral term.

\section{Numerical Valuation of Call options under MEMM}

In this Section we apply our numerical pricing algorithm to the valuation of European call option under the MEMM. The simulated prices are contrasted with those obtained from the Black \& Scholes formula based on a geometric Brownian motion with comparable parameters. Further, we study the volatility smile in the context of our pricing approach.

We have proposed schemes to handle the partial differential equations for both integroPDEs in the coupled system. The finite difference schemes described above have been implemented in $\mathrm{C}++$ and run on designated simulation servers. The integrand is dependent on both $s$ and $y$ and hence we need to do an integration for every grid point and time step, leading to a significant increase in the simulation load compared to ordinary PDEs. Solving on a $100 \times 100$ grid with 35 extra points in the $y$-direction and 50 time steps executes in about 11 seconds. We observed that making the grid finer in the $y$-variable gave a super-linear increase in the simulation time.

Assume that the squared volatility have a stationary distribution being inverse Gaussian $I G(\gamma, \delta)$. As noted by Barndorff-Nielsen and Shephard [6], this choice of volatility process implies that the log-returns of $S(t)$ become approximately normal inverse Gaussian distributed. The Lévy measure of the subordinator $L(t)$ is then

$$
\ell(\mathrm{d} z)=\frac{\delta}{2 \sqrt{2 \pi}} z^{-3 / 2}(1+\gamma z) \exp \left(-\frac{1}{2} \gamma z\right) \mathrm{d} z .
$$

Below follows some results from the simulations, starting with the solution to (2.7). For the volatility process we use the same parameters as Nicolato and Venardos [15]:

$$
\lambda=2.4958, \gamma=11.98, \delta=0.0872 .
$$




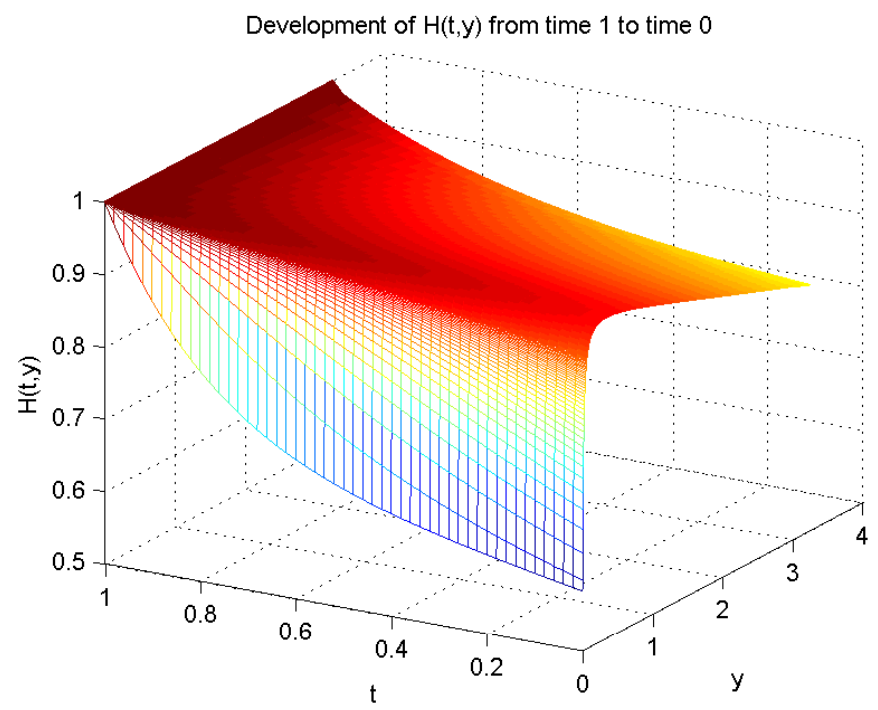

Figure 1. Simulations of $H(t, y)$, with the parameter values from Nicolato and Venardos [15].

For the option we let the strike be $K=200$, and suppose zero interest rate, $r=0$. We let the constants in the market model (2.1) be

$$
\mu=0.05, \beta=0.5 \text {. }
$$

In most examples below we work with a grid of size $251 \times 201$ points, except for the comparison with the Black \& Scholes prices, where we chose a much finer grid of $1501 \times 401$ points.

Since $H(t, y)$ occurs as a measure change in the integral of the partial differential equation (2.13), we need to simulate it before we can solve for the option price. Figure 1 shows a plot of the function $H(t, y)$ based on the chosen parameters.

In Figures 2-4 we show the resulting option prices as a function of $t, s$ and $y$. Remark that in neither of the figures we have plotted the whole solution area, which was $(s, y) \in[0600] \times[01]$. In Fig. 2 we have fixed $y$ to be $y=0.1528$, while in Fig. 4 the asset price is set to $s=340$. Not surprisingly, we see in Fig. 2 that the shape of the price surface as a function of $s$ and $t$ resembles quite well the Black \& Scholes price surface. However, an interesting question is now to what extent the two pricing methodologies are differing.

To get a better picture of how the MEMM prices relate to prices from the Black \& Scholes formula we need first to determine what volatility we should use in the Black \& Scholes formula. We suggest the following procedure: We find the expectation of $Y_{t}$ for the stationary distribution and let the squared volatility in Black \& Scholes, $\sigma_{B S}^{2}$, be equal to this expectation. We then choose the starting value $y$ for the process $Y_{t}$ to be the point closest to $\sigma_{B S}^{2}$ in the grid. This means that we compare Black \& Scholes prices with constant volatility to our indifference prices which have a volatility fluctuating around this constant level. As we see in Fig. 5 the difference has a "W-shape" where MEMM prices are lower at-the-money and higher for in-the-money and out-of-the money. This reflects the Black \& Scholes model's inability to capture the risk of large price movements. The form of the difference is similar to results from Eberlein [9], who prices options in an exponential Lévy model with the 


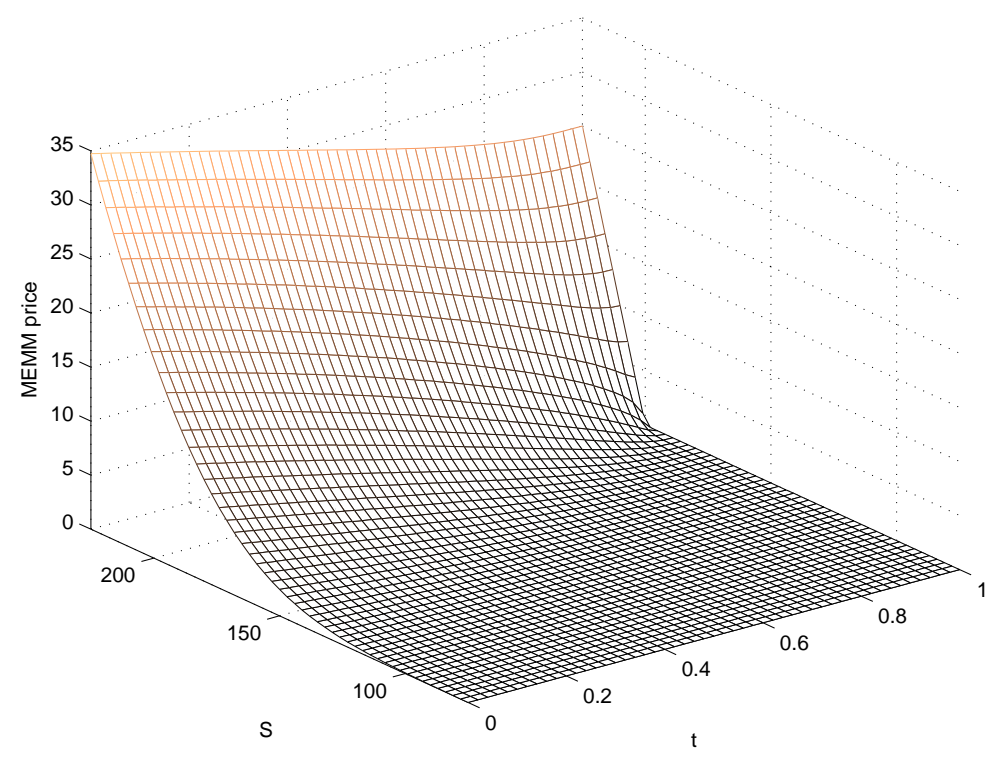

Figure 2. Option prices under the MEMM as a function of time and underlying asset price.

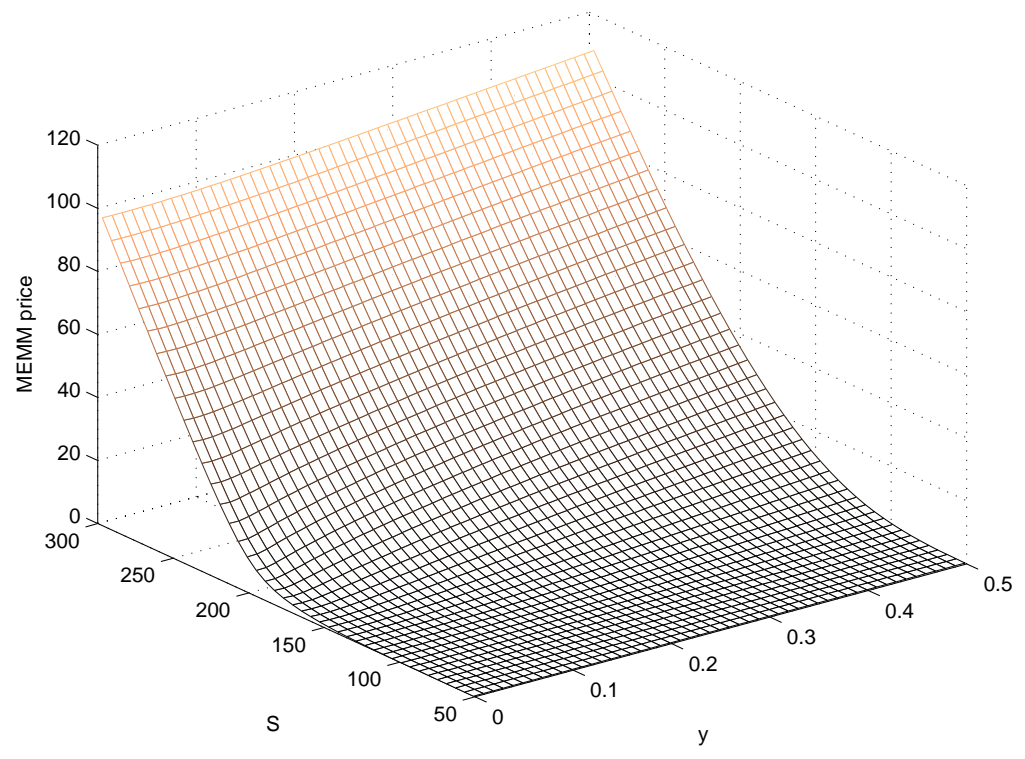

Figure 3. Option prices under the MEMM as a function of the underlying asset price and squared volatility level. 


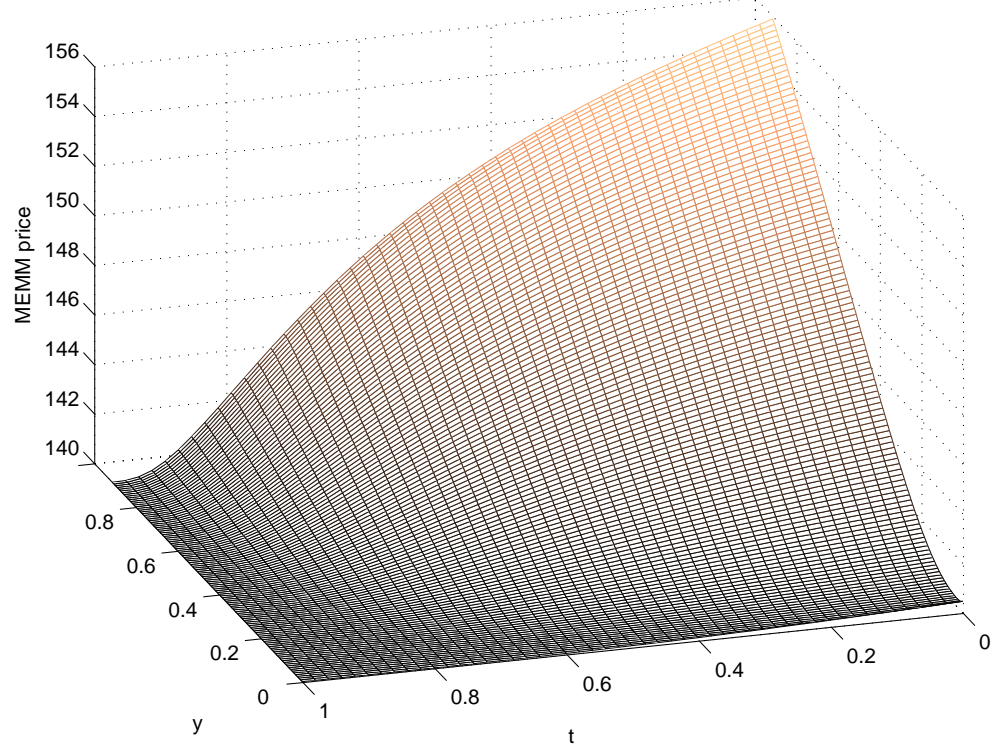

Figure 4. Option prices under the MEMM as a function of the squared volatility level and time.

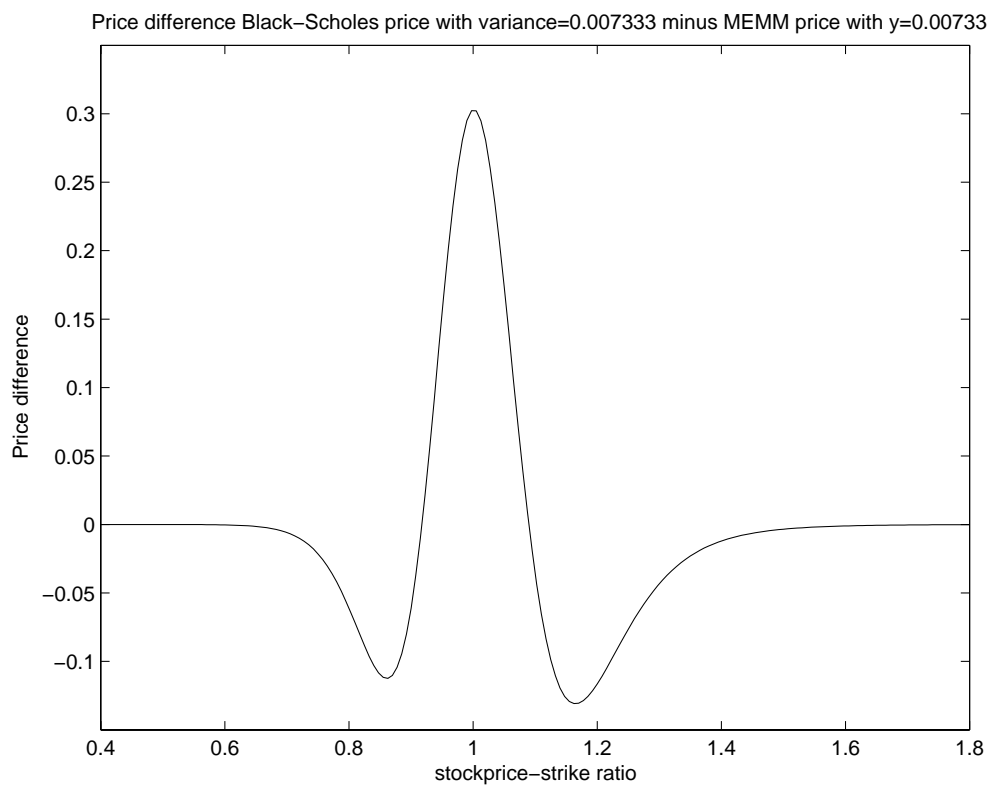

Figure 5. Plot of the difference between the Black \& Scholes price and the MEMM price. 


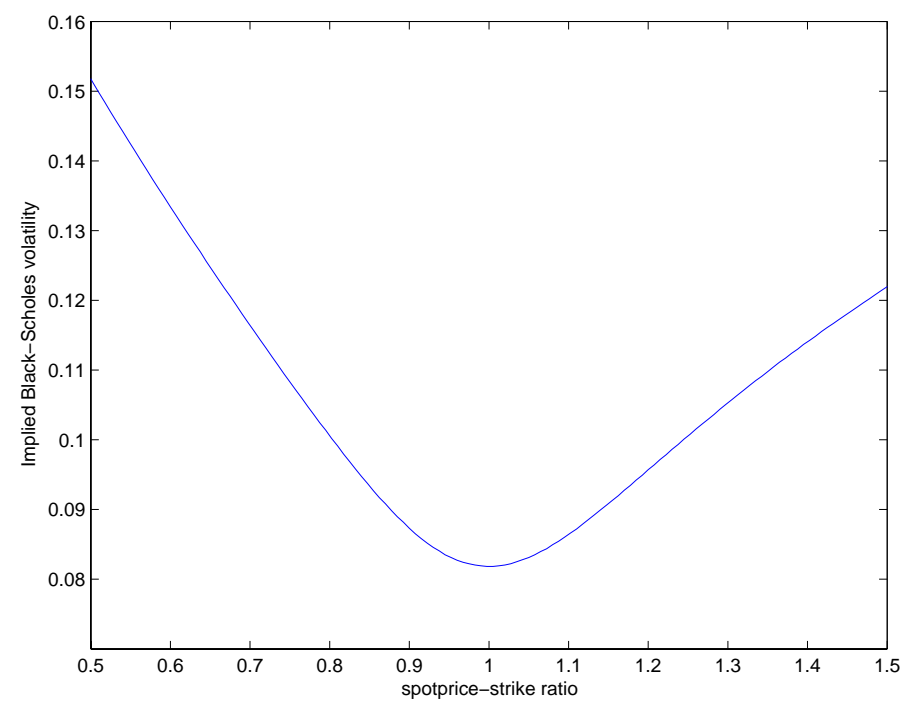

Figure 6. Plot of the implied Black-Scholes volatility produced by the MEMM prices.

hyperbolic distribution based on structure preserving risk-neutral measure obtained through Esscher transformation.

Let us consider the implied volatility yielded by our MEMM prices. We simulated prices for a range of strikes and calculated the implied Black \& Scholes price given by these, assuming the spot price is $s=200$. As we see in Figure 6 we get a skewed volatility smile.

5.1. Pricing of the jump risk under MEMM. An interesting question is how the jump risk is priced under the MEMM. We know that the MEMM is transforming the jump measure of the subordinator $L$ by a ratio of the function $H$. Thus, small and big jumps are rescaled according to the time and state-dependent ratio $H(t, y+z) / H(t, y)$. We have done some numerical tests demonstrating how the jump measure is re-distributed under the MEMM. In Figure 7 we have plotted the ratio for the parameters chosen in the numerical examples above for two different values of $\mathrm{y}$. We have fixed $t=1$ and let $y=0.0317$ (left) and $y=0.1650$ (right). We see that smaller jumps are scaled up, before the ratio dampens the bigger jumps. For small values of $y$ the left pictures indicates that all jumps up to quite large jump sizes are scaled up, while in the right picture jumps with size larger than 0.7 will be scaled down. In fact, we observe here that the large jumps are less influential under the MEMM than under the objective measure, showing that the MEMM puts less value to these. The positive jump risk price is assigned to small jumps and we note that for very small $y$ this upscaling is substantial, increasing towards infinity as we approach zero from the right.

5.2. A discussion of convergence. We end this Section with a few words on convergence of our numerical procedure. In the present paper we have not considered this question from a theoretical point of view, but refer the reader to the works by Amadori [1], Amadori, Karlsen and La Chioma [2] and Jakobsen and Karlsen [12], where convergence is analyzed for integroPDEs similar to ours. In order to justify that our numerical solution of $\Lambda$ indeed converges, 

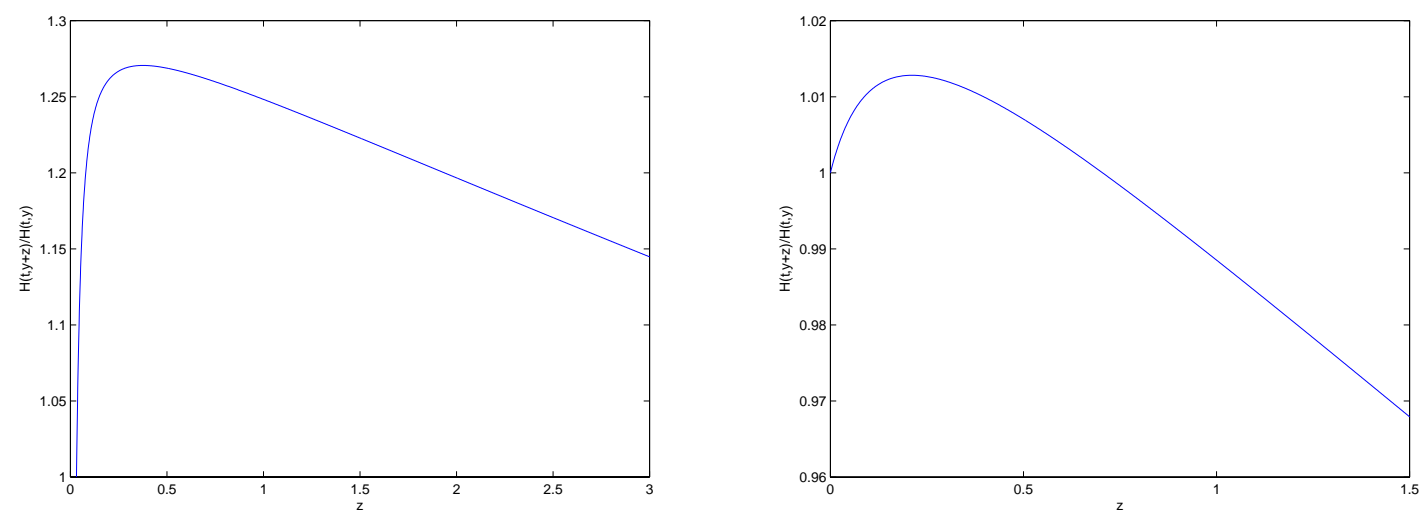

Figure 7. Plots of the ratio $H(t, y+z) / H(t, y)$, illustrating the scaling of the jumps. Here $y=0.0317$ (left) and $y=0.165$ (right).

we have tested the algorithm with step-wise refining of the grid. The relative distance of the resulting numerical solution with respect to the obtained one for the finest grid is shown in Figure 8. We see from this plot that the relative error decreases, indicating that we have convergence. It is the goal in future studies to analyze the convergence and stability of the numerical scheme from a mathematical point of view.

\section{REFERENCES}

[1] A. L. Amadori (2001). Differential and integro-differential nonlinear equations of degenerate parabolic type arising in the pricing of derivatives in incomplete market. PhD thesis, University of Roma I - La Sapienza.

[2] A. L. Amadori, K. H. Karlsen, and C. La Chioma (2004). Nonlinear degenerate integro-partial differential evolution equations related to geometric Lévy processes and applications to backward stochastic differential equations. Stoch. Stoch. Rep., 76(2), pp. 147-177.

[3] G. Barles (1997). Convergence of numerical schemes for degenerate parabolic equations arising in finance theory. In L. Rogers and D. Talay, editors, Numerical Methods in Finance. Cambridge University Press, Cambridge.

[4] G. Barles, C. Daher, and M. Romano (1995). Convergence of numerical schemes for parabolic equations arising in finance theory. Math. Models Methods Appl. Sc., 5(1), pp. 125-143.

[5] G. Barles and P. Souganidis (1991). Convergence of approximation schemes for fully nonlinear second order equations. Asymp. Anal., 4, pp. 271-283.

[6] O. E. Barndorff-Nielsen and N. Shephard (2001). Non-Gaussian Ornstein-Uhlenbeck-based models and some of their uses in financial economics. J. Roy. Stat. Soc. A, 63, pp. 167-241.

[7] F. E. Benth and T. Meyer-Brandis (2005). The density process of the minimal entropy martingale measure in a stochastic volatility model with jumps. Finance Stoch., 9(4), pp. 563-575.

[8] J. Bertoin (1998). Lévy Processes. Cambridge University Press, Cambridge.

[9] E. Eberlein (2001). Application of Generalized Hyperbolic Lévy motions to Finance. In O. E. BarndorffNielsen, T. Mikosch, and S. I. Resnick, editors, Lévy processes, Theory and Applications, pp. 319-336. Birkhäuser, Boston.

[10] S. Gudonov (1959). Finite difference methods for numerical computations of discontinuous solutions of the equations of fluid dynamics. Matematiceskij Sbornik, 47, pp. 271-306.

[11] J. C. Hull and A. White (1987). The pricing of options on assets with stochastic volatility. J. Finance, 42, pp. $281-300$.

[12] E. R. Jakobsen and K. H. Karlsen (2004). A 'maximum principle for semicontinuous functions' applicable to integro-partial differential equations. To Appear in: Nonlin. Diff. Eq. Appl. 


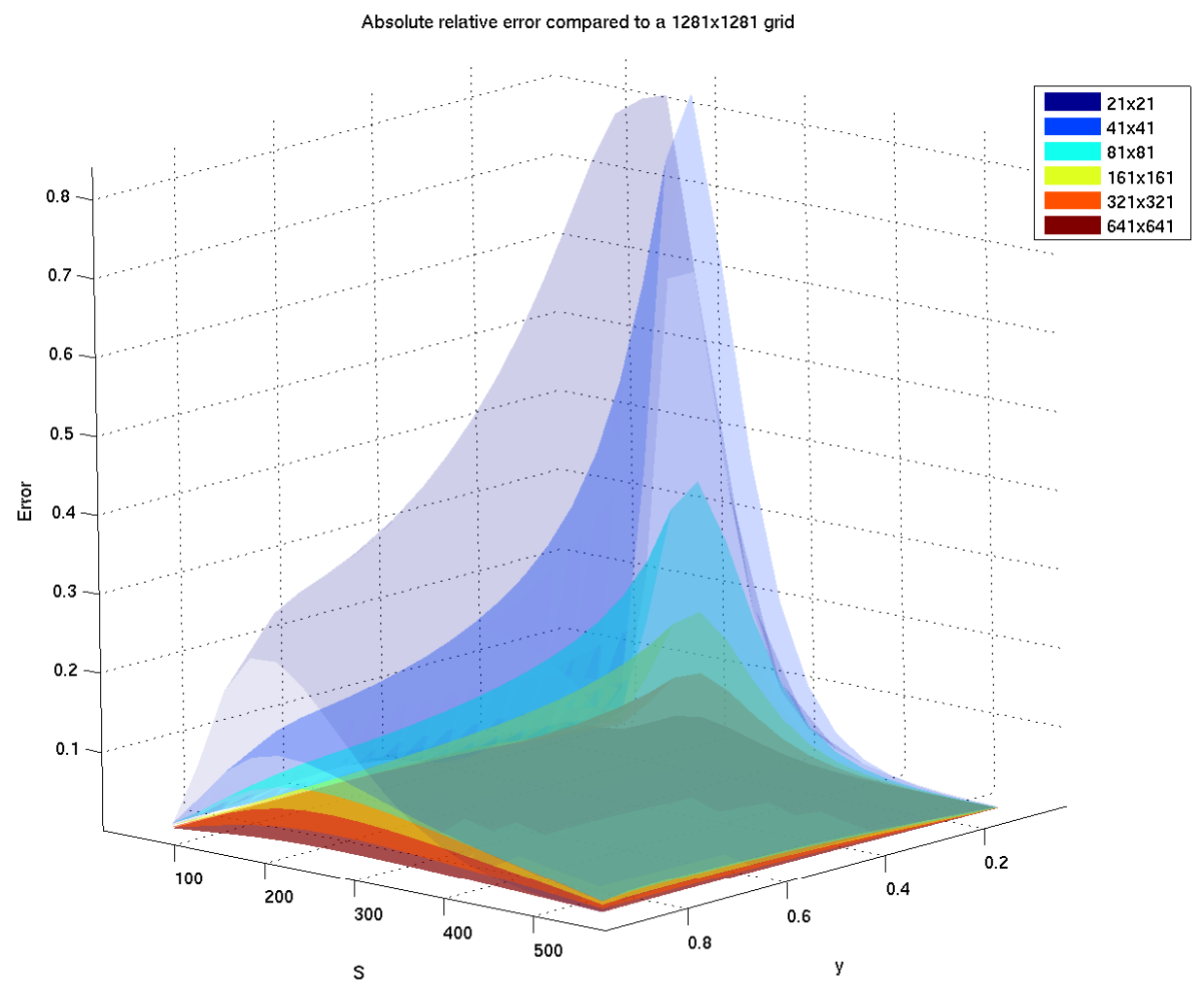

Figure 8. Plot of the relative error with respect to the solution obtained on the finest grid.

[13] D. Kröner (1997). Numerical schemes for conservation laws. John Wiley \& Sons and B.G. Teubner Publisher, Chichester.

[14] A. L. Lewis (2000). Option valuation under stochastic volatility. Finance Press, Newport Beach, California.

[15] E. Nicolato and E. Venardos (2003). Option pricing in stochastic volatility models of the OrnsteinUhlenbeck type. Math. Finance, 13(4), pp. 445-466.

[16] T. Rheinländer and G. Steiger (2005). The minimal martingale measure for general BarndorffNielsen/Shephard models. Preprint, London School of Economics.

[17] G. Strang (1968). On the construction and comparison of difference schemes. SIAM J. Num. Anal., 5, pp. 506-517. 
(Fred Espen Benth)

Centre of Mathematics for Applications

Department of Mathematics

UNIVERSITY OF OSLO

P.O. Box 1053, BLINDERN

N-0316 Oslo, Norway

AND

Agder University College

SChoOl of Management

SERVICEBOKS 422

N-4604 Kristiansand, NorWAy

E-mail address: fredb@math.uio.no

$U R L:$ http://folk. uio.no/fredb

(Martin Groth)

Centre of Mathematics for Applications

Department of Mathematics

UNIVERSITY OF OSLO

P.O. Box 1053, BLINDERN

N-0316 Oslo, Norway

E-mail address: martijg@math.uio.no

$U R L:$ http://folk.uio.no/martijg 